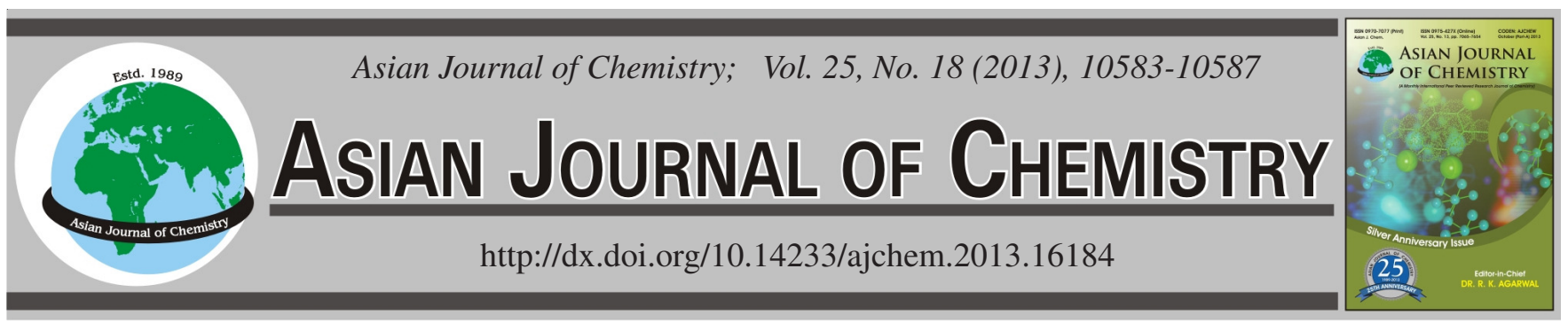

\title{
Antiinflammatory Evaluation and Docking Studies of Some New Thienopyrimidines
}

\author{
Deb Pran Kishore ${ }^{1, *}$, Raghuprasad Maillabaram $^{2, *}$, A. Raghuram Rao ${ }^{3}$ and P. Mallikarjuna Rao ${ }^{1}$
}

${ }^{1}$ Department of Pharmaceutical Chemistry, School of Pharmacy, International Medical University, 126, Jalan Jalil Perkasa 19, Bukit Jalil, 57000 Kuala Lumpur, Malaysia

${ }^{2}$ Pharmaceutical Chemistry Division, Sri Vishnu College of Pharmacy, Vishnupur, Bhimavaram-534 201, India

${ }^{3}$ Medicinal Chemistry Division, University College of Pharmaceutical Sciences, Kakatiya University, Warangal-506 009, India

*Corresponding authors: E-mail: prankishore@imu.edu.my, raghumrp@ rediffmail.com

\begin{abstract}
A series of some new 6-substituted-2,3,4-trihydropyrimido[1,2-c]9,10,11,12-tetrahydrobenzo[ $b]$ thieno[3,2-e]pyrimidines (1-6) have been evaluated in silico (docking studies) to recognize their hypothetical binding motif with the cyclooxygenase isoenzyme (COX-2) employing GLIDE software (Schrodinger Inc.) and in vivo (rat paw edema) for their antiinflammatory activities. The compound 6 with pyridyl substitution at the $6^{\text {th }}$ position of the thienopyrimidine moiety was found to form significant $\mathrm{H}$-bonding interaction with crucial amino acid residue Arg 120 at a distance of $3 \AA$ and exhibited good antiinflammatory activity [around $87 \%$ of the standard: indomethacin]. The binding mode of the thienopyrimidines compounds have been proposed based on the docking studies. Further, the predicted ADME properties of all the tested compounds were found to be in the ranges as predicted by QikProp for $95 \%$ of known oral drugs and also satisfy the Lipinski's rule of five.
\end{abstract}

Key Words: Thienopyrimidines, Antiinflammatory activity, COX-2 docking studies, in silico ADME prediction.

\section{INTRODUCTION}

The nonsteroidal antiinflammatory drugs (NSAIDs) are important therapeutic agents used for the treatment of pain and inflammation, act by reducing the production of proinflammatory prostaglandins (PGs) at the sites of injury via cyclooxygenase-2 (COX-2) inhibition ${ }^{1,2}$. It has been observed that COX isoenzymes exist in two different isoforms, a constitutive form (COX-1) and an inducible form (COX-2) ${ }^{3}$. The constitutive COX-1 isozyme is important for the maintenance of various physiological functions such as cytoprotection of gastric mucosa, regulation of renal blood flow and platelet aggregation ${ }^{4}$. In contrast, the expression of COX-2 isozyme is rapidly induced by stimuli such as mitogenes and oncogenes, growth factors, hormones and disorders of water-electrolyte homeostasis linking its involvement to pathological processes such as inflammation and various cancer types ${ }^{5-7}$. The side effects associated with most of the NSAIDs such as gastrointestinal ulcer and renal function suppression are due to the inhibition of COX-1 pathway ${ }^{1,2,8}$. Thus the success of NSAIDs in treatment of various inflammatory disorders depends on the selective inhibition of COX-2 over COX-1 isoenzyme. Failure of some COXIBs such as rofecoxib due to its adverse cardiovascular side effects ${ }^{9}$ imposed a great challenge to the researchers to explore and evaluate alternative templates with selective COX-2 inhibitory activity ${ }^{10}$. Recently, a series of $8 /$ 10-trifluo-romethyl-substituted-imidazo[1,2-c] quinazolines ${ }^{2}$, 3-alkoxy-4-methanesulfonamido acetophenone derivatives1 cycloalkyl/aryl-3,4,5-trimethylgallates ${ }^{10}$ have been reported from our laboratory as potent antiinflammatory agents. Earlier, a series of novel 6-substituted-2,3,4-trihydropyrimido[1,2$c] 9,10,11,12$-tetrahydrobenzo[b]thieno[3,2-e]pyrimidines (1-6) have been synthesized and reported as antibacterial agents ${ }^{11}$. Interestingly, these compounds (1-6) also exhibited good antiinflammatory activity based on in silico docking studies using the COX-2 receptor. Hence it was taught of interest to test these compounds (1-6) for their in vivo antiinflammatory activity. Further, we propose the molecular interactions and the binding mode of these target compounds (1-6) using COX2 isoenzyme based on in silico docking studies.

\section{EXPERIMENTAL}

Molecular modeling investigations were carried out using Dell Precision work station T3400 running Intel Core2 Duo Processor, 4GB RAM, 250 GB hard disk and NVidia Quodro FX 4500 graphics card. Maestro 9.4, GLIDE v5.9 XP docking program (Schrodinger Inc.) was employed for the docking studies $^{12,13}$. 
Preparation of protein: PDB structure (www.rcsb.org) 1CX2 (Crystal structure of COX-2) was downloaded, refined and prepared using Schrodinger protein preparation wizard tool (Glide), which performs the following steps: assigning of bond orders, addition of hydrogens, optimization of hydrogen bonds by flipping amino side chains, correction of charges and minimization of the protein complex. All the bound water molecules, ligands and cofactors were removed (preprocess) from the proteins which were taken in.mae format. The tool neutralized the side chains that are not close to the binding cavity and do not participate in salt bridges. This step is then followed by restrained minimization of co-crystallized complex, which reorients side chain hydroxyl groups and alleviates potential steric clashes. The complex obtained was minimized using OPLS_2005 force field ${ }^{14}$ with Polack-Ribiere Conjugate Gradient (PRCG) algorithm. The minimization was terminated either completion of 5,000 steps (or) after the energy gradient converged below $0.05 \mathrm{kcal} / \mathrm{mol}$.

Preparation of ligands: Structures of the ligands (1-6) were sketched using built panel of maestro and taken in mae format. LigPrep is a utility of schrodinger software suit that combines tools for generating 3D structures from 1D (Smiles) and 2D (SDF) representation, searching for tautomers, steric isomers and perform a geometry minimization of the ligands. Molecular Mechanics Force Fields (OPLS_2005) with default settings were employed for the ligand minimization.

Docking studies: Docking studies were carried out using the above mentioned prepared protein (PDB: 1CX2) and ligands (1-6), by employing Glide XP docking program (Schrodinger Inc.) following the reported procedure ${ }^{12,13}$.

Prediction of absorption, distribution, metabolism and excretion properties: The QikProp module of Schrodinger is a quick, accurate, easy-to-use absorption, distribution, metabolism and excretion (ADME) prediction program design to produce certain descriptors related to ADME. QikProp predicts physically significant descriptors and pharmaceutically relevant properties of organic molecules, either individually or in batches. QikProp has two modes: normal mode and fast mode. In fast mode, certain time-consuming calculations are omitted, some properties are not predicted and some have different values. In the present study, QikProp was run in normal processing mode with default options ${ }^{15}$. To set up the calculation, pose viewer file (generated after docking with Glide) was used to consider the receptor and source of ligands. After choosing the receptor and ligands, by using pose viewer file (pv.maegz), the program QikProp that generate the descriptors were run with default options that were chosen to produce reasonable descriptors. The selected properties that are known to influence metabolism, cell permeation and bioavailability are presented in Table- 2 .

Pharmacological evaluation: Antiinflammatory activitycarrageenan-induced rat paw edema assay: The in vivo antiinflammatory screening for all the test compounds (1-6) was performed by using the functional model of carrageenaninduced rat paw edema and is presented as the percentage inhibition of edema at the right hind paw in comparison to the control (Table-1). Carrageenan-induced edema is a nonspecific inflammation but is highly sensitive to NSAIDs. Indomethacin, a potent NSAID was used as a reference standard.
COX-2-mediated increase in prostaglandin E2 (PG-E2) production contributes to the severity of the inflammatory and pain responses in this model. Wistar rats (female) weighing between 190 and $260 \mathrm{~g}$, obtained from Central Animal House, Panjab University, Chandigarh, India and were used in the present study. Animals were kept in wire-mesh cages and maintained under constant environmental food and water (ad libitum), in a constant lightedark cycle. During the course of experiment, the general behavior of animals was normal. All the experimental protocols were approved by the Institutional Animal Ethical Committee (IACE) and experiments were conducted in accordance with the standard guidelines. Carrageenan and indomethacin were procured from Sigma Chemical, Co. For statistical analysis we have used GraphPad Prism 3.0 version $^{16}$.

The target compounds (1-6) were tested for their antiinflammatory activity against carrageenan-induced paw edema at dose of $10 \mathrm{mg} / \mathrm{kg}$. The percentage inhibition of edema was calculated using the formula given below:

$$
\frac{\mathrm{V}_{\mathrm{c}}-\mathrm{V}_{\mathrm{t}}}{\mathrm{V}_{\mathrm{c}}} \times 100
$$

where $V_{c}$ is the increase in paw volume of control (in the absence of test compound) and $\mathrm{V}_{\mathrm{t}}$ is the increase in paw volume after administration of the test compound.

The given data is of five animals (female wistar rats) per group and divided into eight (8) groups. Group-1 referred as control (animals received carrageenan along with the vehicle [0.5\% carboxymethylcellulose (CMC)] and Group-2 received standard reference (indomethacin) along with the vehicle prior to the administration of carrageenan. Group-3, 4, 5, 6, 7 and 8 received the test compounds (1-6) respectively $1 \mathrm{~h}$ prior to the administration of carrageenan. All the test compounds were suspended in $0.5 \%$ of CMC and administered orally $(10 \mathrm{mg} / \mathrm{kg})$ $60 \mathrm{~min}$ prior to the injection of $0.1 \mathrm{~mL}$ of freshly prepared carrageenan $(1 \%)$ in physiological solution $(154 \mathrm{mM} \mathrm{NaCl})$ into the sub-planter tissue of hind paw of each rat. The equivalent volume of carrageenan $(1 \%)$ in physiological solution was injected into hind paw of the control. The volume was measured three times using water plethysmometer prior to the administration of carrageenan, $2 \mathrm{~h}$ and $4 \mathrm{~h}$ after the injection. The increase in volume of the paw was adopted as a measure of edema ${ }^{17}$. The antiedematous effects of the compounds were estimated as percentage inhibition of the induced inflammation in comparison with control. Statistical analysis was carried out using a one-way analysis of variance (ANOVA). In all cases, post-hoc comparisons of the means of individual groups were performed using Tukey's test. A significance level of $\mathrm{p}<$ 0.001 denoted significance in all cases.

\section{RESULTS AND DISCUSSION}

The target compounds 6-substituted 2,3,4-trihydropyrimido[1,2-c]9,10,11,12-tetrahydrobenzo[b]thieno[3,2-e]pyrimidines (1-6) were evaluated in silico (docking) to recognize their hypothetical binding mode as well as affinity using the X-ray crystal structure of COX-2 (PDB ID: 1CX2). To investigate the ability of molecular docking to reproduce an experimentally observed ligand-binding mode, the co- 


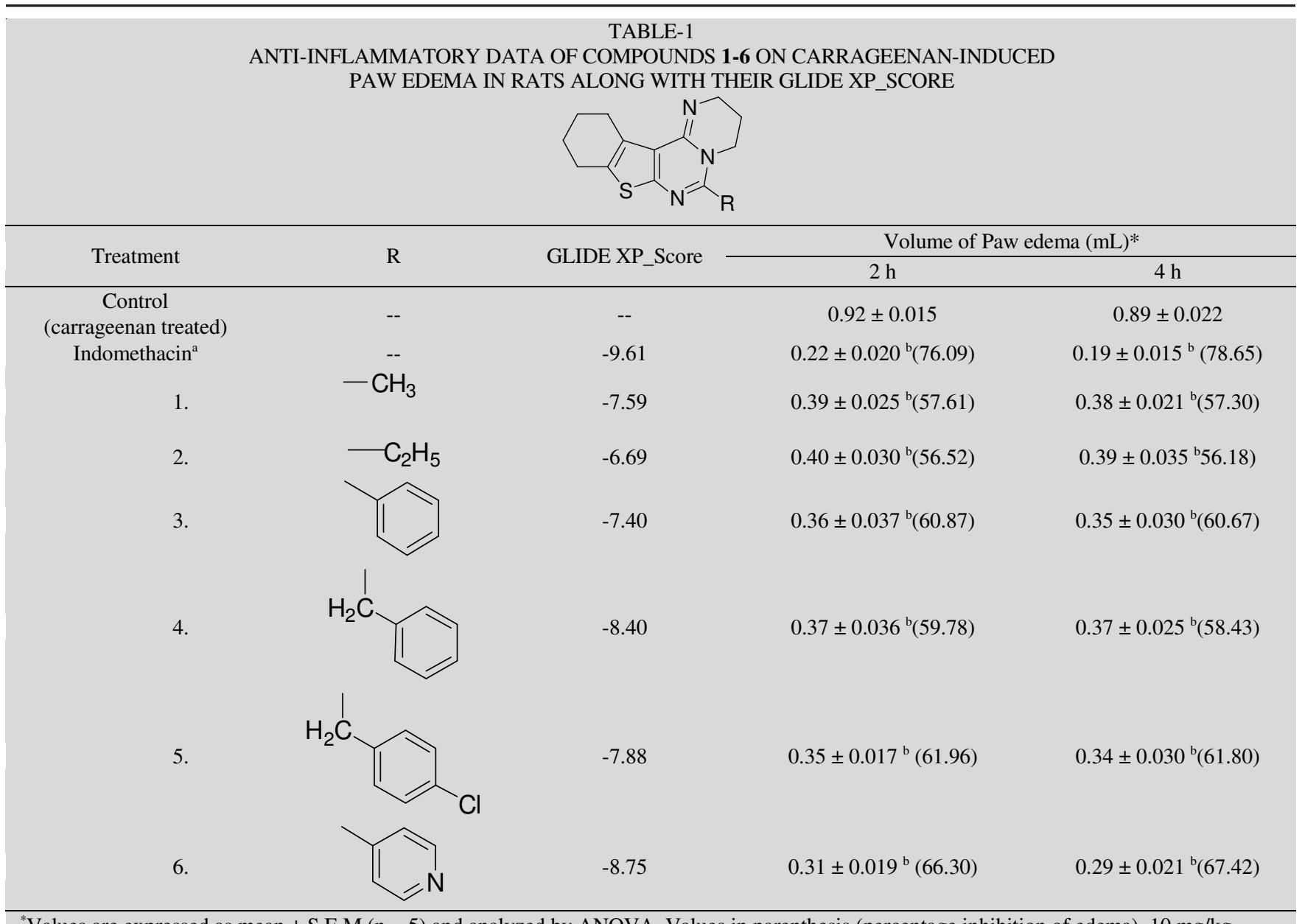

"Values are expressed as mean \pm S.E.M ( $\mathrm{n}=5)$ and analyzed by ANOVA. Values in parenthesis (percentage inhibition of edema), $10 \mathrm{mg} / \mathrm{kg}$

${ }^{\mathrm{a}}$ Reference standard, ${ }^{\mathrm{b}}$ Different from carragenan group $(\mathrm{p}<0.001)$

TABLE-2

PROPERTIES OF TESTED COMPOUNDS (1-6) CALCULATED BY QikProp

\begin{tabular}{|c|c|c|c|c|c|c|}
\hline Comp. No. & $\mathrm{QP} \log \mathrm{Po} / \mathrm{w}^{\mathrm{a}}$ & $\mathrm{QP} \log \mathrm{S}^{\mathrm{b}}$ & $\mathrm{QPPCaco}^{\mathrm{c}}$ & $\#$ metab ${ }^{d}$ & $\begin{array}{l}\text { Percent Human } \\
\text { Oral Absorption }^{\mathrm{e}}\end{array}$ & Rule Of Five ${ }^{f}$ \\
\hline SC-558 & 3.571 & -6.063 & 371.676 & 0 & 93.856 & 0 \\
\hline Indomethacin & 4.258 & -5.081 & 169.265 & 3 & 91.764 & 0 \\
\hline 1. & 3.667 & -4.587 & 5740.881 & 4 & 100 & 0 \\
\hline 2. & 4.111 & -5.053 & 7461.526 & 4 & 100 & 0 \\
\hline 3. & 4.017 & -5.184 & 3711.061 & 4 & 100 & 1 \\
\hline 4. & 5.522 & -6.48 & 7824.774 & 4 & 100 & 1 \\
\hline 6. & 3.989 & -4.894 & 4230.635 & 3 & 100 & 0 \\
\hline
\end{tabular}

${ }^{a}$ Predicted log of the octanol/water partition coefficient, range $95 \%$ of drugs (-2-6.5), ${ }^{b}$ Predicted log of aqueous solubility S (mol/L), range $95 \%$ of drugs (-6.5-0.5), ${ }^{\mathrm{c}} \mathrm{Caco} 2$ cell permeability in $\mathrm{nm} / \mathrm{s}$, range $95 \%$ of drugs $(<25$ poor $>500$ great $)$. Caco-2 cells are a model for the gut-blood barrier. QikProp predictions are for non-active transport, ${ }^{\mathrm{d}}$ Number of likely metabolic reactions. Range $95 \%$ of drugs $(1-8)$, ${ }^{\mathrm{e}}$ Predicted human oral

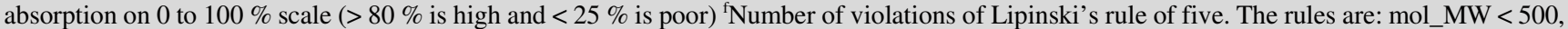
$\mathrm{QPlogPo} / \mathrm{w}<5$, donor HB $\leq 5$, accptHB $\leq 10$. Compounds that satisfy these rules are considered druglike. (The "five" refers to the limits, which are multiples of 5)

crystallized ligand SC-558 (a selective COX-2 inhibitor) has been used as reference ligand (Fig. 1a). It was docked back into its binding site (Fig. 1a) of the crystal structure of the COX-2 using GLIDE docking program (Schrodinger Inc.). The top docked conformations (poses) closely resembled the co-crystallized conformation with a root-mean-square deviation (RMSD) $0.50-0.90 \AA$ of nonhydrogen atomic positions of the ligand (SC-558). The experimental binding mode of SC-558 was reproduced and as shown in the Fig. 1a. The trifluoromethyl group $\left(-\mathrm{CF}_{3}\right)$ formed strong hydrogen bonding interactions with the exocyclic $\mathrm{NH}_{2}$ group of Arg120 at a distance of 2.087 $\AA$. The same group $\left(-\mathrm{CF}_{3}\right)$ was also found to be located in the vicinity of Leu531, Val116, Val349, Leu359 and Tyr355. The sulphonamide $-\mathrm{NH}_{2}$ was found to form hydrogen bonding with the nitrogen atom of imidazole ring of His90 and oxygen atom of Ser353 at a distance of $2.505 \AA$ and $3.19485 \AA$ respectively, whereas sulphonamide oxygen $(=\mathrm{O})$ formed hydrogen bond with the NH of Arg513. Pyrazole ring of SC-558 is found to be located within the vicinity of Tyr355 and Val349. Bromophenyl moiety was surrounded by Gly526, Val523 and Ala527. 
These residues were identified as crucial and involved in the binding which perhaps contributing to the selectivity of the ligand.

Earlier from the docking studies of the indomethacin, a nonselective $\mathrm{COX}-1 / 2$ inhibitor, it has been observed that the carboxylate ion formed a salt bridge with the Arg120 in COX2 isoenzyme which gives a more generalized anchoring point for all the classical NSAIDs thus limiting their selectivity due to curtailed freedom of movement of the ligand ${ }^{2}$. The present docking study also revealed the similar observation (Fig. 1b).

Interestingly, all the target compounds (1-6) docked well into the binding pocket of COX-2 (Fig. 1c). In particular the cyclic $\mathrm{N}$-atom of the pyridyl group attached to the $6^{\text {th }}$ position of thienopyrimidine moiety of the most active compound 6 formed a significant $\mathrm{H}$-bond interaction with the crucial amino acid residue Arg120 at a distance of 3.008 A (Fig. 1d) similar to trifluoromethyl group $\left(-\mathrm{CF}_{3}\right)$ of $\mathbf{S C 5 5 8}$. The pyridyl group was also found to be located in the vicinity of Leu531, Val116, Val349, Leu359 and Tyr355. The tricyclic nucleus 9,10,11,12tetrahydrobenzo $[b]$ thieno[3,2-e]pyrimidine of all the compounds were found to be located well in the binding pocket and aligned with the bromophenyl ring of SC-558. The 2,3,4trihydropyrimido moiety was found to overlap with phenylsulfonamide group in a cavity formed by crucial amino acid residue responsible for COX-2 selectivity as well as affinity such as Phe518, Leu352, Val523, His90, Arg513 and Gln192. The predicted binding energies (GLIDE XP_Score) of the compounds and the corresponding experimental values are also listed in Table-1. Further none of the compounds showed any kind of salt bridge formation like non-selective indomethacin.

Prediction of absorption, distribution, metabolism and excretion properties: The expected ADME properties of the tested compounds were evaluated with QikProp module of Schrodinger (Table 2). The selected properties are known to influence metabolism, cell permeation and bioavailability. Almost all the predicted properties of the tested compounds were in the ranges as predicted by QikProp for $95 \%$ of known oral drugs and also satisfy the Lipinski's rule of five to be considered as drug like potential.
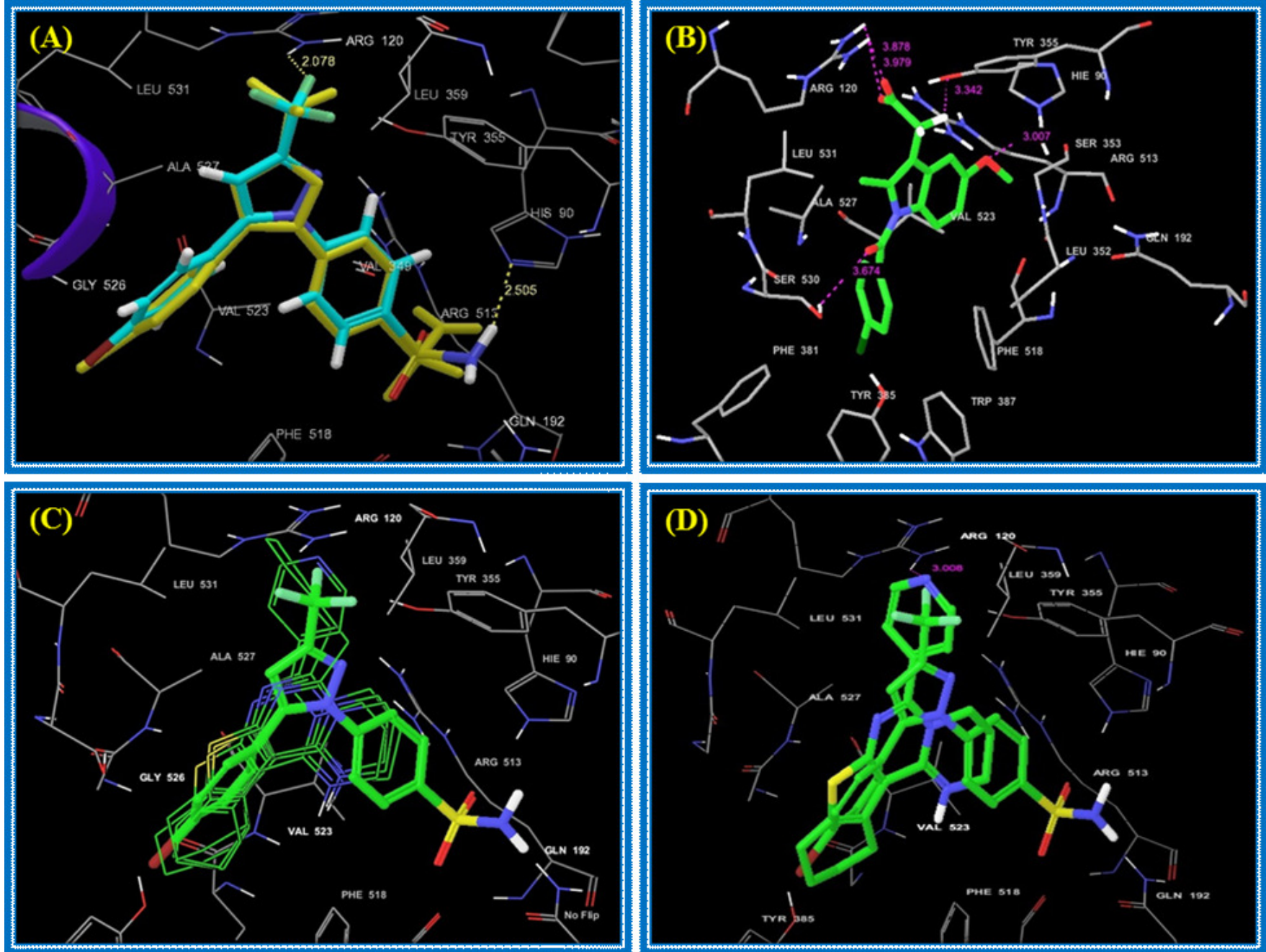

Fig. 1. (a) Superimposition of redocked SC-558 (cyan color) with its original position (yellow color) as co-crystal in the binding site of the crystal structure of COX-2 (PDB ID:1CX2) isoenzyme (RMSD-0.70) showing H-bond interactions with the amino acid residues His90, Arg120, Ser353, and Arg513, respectively (b) Binding orientation of indomethacin within the binding site of COX-2 showing salt bridge formation between the carboxylate ion and $\operatorname{Arg} 120$ (c) Binding orientation of all the docked ligands (1-6) and their superimposition with the co-crystal ligand SC-558 within the binding site of COX-2 (d) Hypothetical binding motif of compound $\mathbf{6}$ superimposed with the co-crystal ligand SC-558 within the binding site of COX-2 showing important H-bonding interaction with the amino acid residues Arg 120 
(A)

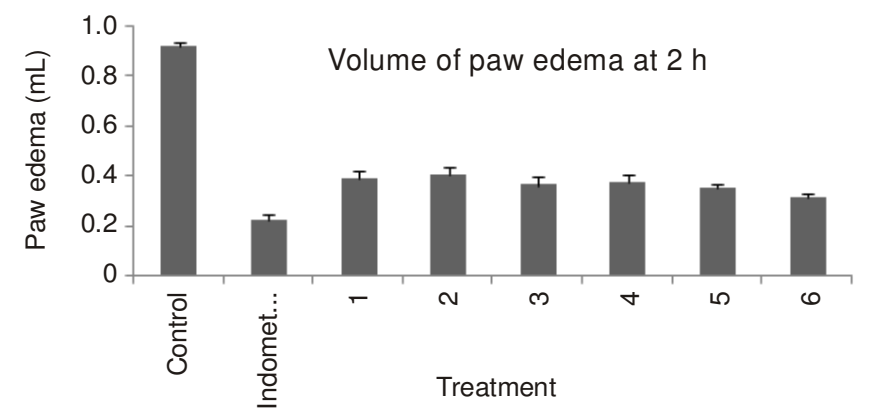

(B)

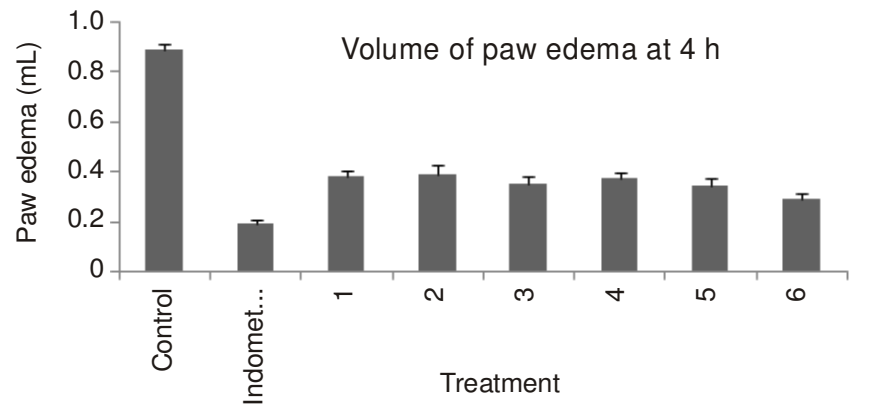

Fig. 2. Treated groups $v s$. paw edema (mL) (a) after $2 \mathrm{~h}(\mathrm{~b})$ after $4 \mathrm{~h}$

Antiinflammatory activity: Carrageenan-induced rat paw edema assay: All the title compounds (1-6) were screened for their in vivo antiinflammatory activity using the carrageenan-induced rat paw edema model and exhibited protection against carrageenan-induced edema (Table-1, Figs. 2a and 2b). The protection ranged up to $66 \%$, while the reference drug (indomethacin) showed $76 \%$ at an equivalent dose. Among all the tested compounds, 6-(2-pyridyl)-2,3,4-trihydro-pyrimido$[1,2-c] 9,10,11,12$-tetrahydrobenzo $[b]$ thieno $[3,2-e]$ pyrimidine (6) showed remarkable antiinflammatory activity compared to other derivatives (1-5). Interestingly, it has been observed that all the target compounds with aromatic/aryl substitution (3-6) at the 6th position of the thieno-pyrimidine moiety showed better antiinflammatory activity as compared to other analogues with alkyl substituent $(\mathbf{1} \& \mathbf{2})$. Further aromatic substituent with electronegative atom at the para position showed enhanced activity as compared to other aromatic analogues as evident from compounds 5 and $\mathbf{6}$. In case of compound 6, N-atom of the pyridyl ring was found to form significant $\mathrm{H}$-bond interaction with crucial amino acid residue Arg120 (3.008 ^) which could be the reason for its enhanced activity as compared to other derivatives. But it could not show significant interaction with amino acid residues like His90, Arg513, Gln192 which are known to be key point of interaction for COX-2 selectivity as well as affinity which could be the reason for its less activity as compared to the reference standard. From the above observation, it could be postulated that further introduction of electronegative substituent at strategic position of the aromatic ring could enhance the antiinflammatory activity.

\section{Conclusion}

In the present study, a series of some new 6-substituted 2,3,4-trihydropyrimido[1,2-c]9,10,11,12-tetrahydrobenzo $[b]$ thieno[3,2-e]pyrimidines (1-6) have been evaluated in silico (docking studies) to recognize their hypothetical binding motif with the cyclooxygenase isoenzyme (COX-2) employing GLIDE software (Schrodinger Inc.) and in vivo (rat paw edema) for their antiinflammatory activities. The compound $\mathbf{6}$ with pyridyl substitution at the $6^{\text {th }}$ position of the thienopyrimidine moiety was found to form significant $\mathrm{H}$-bonding interaction with crucial amino acid residue $\operatorname{Arg} 120$ at a distance of $3.008 \AA$ and exhibited good antiinflammatory activity [around $87 \%$ of the standard: indomethacin]. The binding mode of the title compounds have been proposed based on the docking studies. The predicted ADME properties of all the tested compounds were in the ranges as predicted by QikProp for $95 \%$ of known oral drugs and also satisfy the Lipinski's rule of five which signifies a good absorption and hence, good bioavailability so that the observed differences in bioactivity of these compounds may be attributable to the differences in their chemical structures. Further, evaluation of these active compounds for their in vitro COX-2 binding studies is under progress.

\section{ACKNOWLEDGEMENTS}

One of the authors, P.K.D. are thankful to Pharmaceutical Chemistry Division, School of Pharmacy, IMU for providing the facilities to carry out the docking studies. Authors thankfully acknowledge Dr. Kanwaljit Chopra, Pharmacology Division, University Institute of Pharmaceutical Sciences (UIPS), Panjab University, Chandigarh, India, for valuable help during pharmacological studies. Authors are also thankful to Mr. Raghurangaswamy, Executive Director, Schrodinger for providing the evaluation license (14 June - 16 July, 2013) to carry out the molecular modeling studies.

\section{REFERENCES}

1. A. Bali, R. Ohri and P.K. Deb, Eur. J. Med. Chem., 49, 397 (2012).

2. C. Balakumar, P. Lamba, D. Pran Kishore, B. Lakshminarayana, K. Venkat Rao, K. Rajwinder, A. Raghuram Rao, B. Shireesha and B. Narsaiah, Eur. J. Med. Chem., 45, 4904 (2010).

3. S. Fiorucci, R. Meli, M. Bucci and G. Cirino, Biochem. Pharmacol., 62, 1433 (2001).

4. W.L. Smith and D.L. Dewitt, Adv. Immunol., 62, 167 (1996).

5. K. Shigeru, T. Tetsunari and Y. Ken-ichi, Adv. Clin. Chem., 43, (2007).

6. H.R. Herschman, Biochim. Biophys. Acta, 1299, 125 (1996).

7. T. Kawamori, C.V. Rao, K. Seibert and B.S. Reddy, Cancer Res., 58, 409 (1998).

8. C.E. Eberhart and R.N. Dubois, Gastroenterology, 109, 285 (1995).

9. J.-M. Dogné, C.T. Supuran and D. Pratico, J. Med. Chem., 48, 2251 (2005).

10. M.S. Dhingra, P.K. Deb, R. Chadha, T. Singh and M. Karan, Med. Chem. Res., (2013); DOI: 10.1007/s00044-013-0620-6.

11. M. Raghu Prasad and D. Pran Kishore, Chem. Pharm. Bull., 55, 776 (2007).

12. Maestro version 9.4 and Glide v5.9, Schrödinger, LLC, New York, NY, (2013).

13. R.A. Friesner, J.L. Banks, R.B. Murphy, T.A. Halgren, J.J. Klicic, D.T. Mainz, M.P. Repasky, E.H. Knoll, M. Shelley, J.K. Perry, D.E. Shaw, P. Francis and P.S. Shenkin, J. Med. Chem., 47, 1739 (2004).

14. G.A. Kaminski, R.A. Friesner, J. Tirado-Rives and W.L. Jorgensen, J. Phys. Chem., 105, 6474 (2001).

15. QikProp, version 3.6, Schrödinger, LLC, New York, NY, (2013).

16. GraphPad Software Inc, CA, USA. (2007).

17. C.A. Winter, E.A. Risley and G.W. Nuss, Proc. Soc. Exp. Biol. Med., 111, 544 (1962). 